\title{
Do First Impressions of Autistic Adults Differ Between Autistic and Nonautistic Observers?
}

\author{
Kilee M. DeBrabander, BS, ${ }^{1}$ Kerrianne E. Morrison, PhD, ${ }^{1}$ Desiree R. Jones, BA, ${ }^{1}$ Daniel J. Faso, PhD, ${ }^{2}$ \\ Mike Chmielewski, $\mathrm{PhD}{ }^{3}$ and Noah J. Sasson, $\mathrm{PhD}^{1}$
}

\begin{abstract}
Background: Autistic adults receive unfavorable first impressions from typically developing (TD) adults, but these impressions improve when TD adults are made aware of their diagnosis. It remains unclear, however, how autistic adults form first impressions of other autistic adults, and whether their impressions are similarly affected by diagnostic awareness.

Methods: In this study, 32 autistic and 32 TD adults viewed brief videos of 20 TD and 20 autistic adults presented either with or without their diagnostic status and rated them on character traits and their interest in interacting with them in the future.

Results: Findings indicated that autistic raters shared the TD tendency to evaluate autistic adults less favorably than TD adults, but these judgments did not reduce their social interest for interacting with autistic adults as they did for TD raters. Furthermore, informing raters of the diagnostic status of autistic adults did not improve first impressions for autistic raters as they did for TD raters, suggesting that autistic raters either already inferred their autism status when no diagnosis was provided or their impression formation is less affected by awareness of a person's diagnosis.

Conclusions: Collectively, these results demonstrate that autistic observers make trait inferences about autistic adults comparable with those made by TD observers-suggesting a similar sensitivity to perceiving and interpreting social signifiers that differ between TD and autistic presentation styles-but unlike their TD counterparts, these trait judgments are not perceived as an impediment to subsequent social interaction and are relatively consistent regardless of diagnostic disclosure.
\end{abstract}

Keywords: first impressions, autism, diagnostic disclosure

\section{Lay Summary}

Why was this study done?

Typically developing (TD) adults often form negative first impressions of autistic adults and report less of a desire to interact with them. These biases affect the social experiences of autistic adults and can contribute to their social disability. More optimistically, however, first impressions of autistic adults improve when TD adults are more knowledgeable about autism and are made aware of their diagnostic status, suggesting that familiarity and understanding can promote acceptance of autistic differences. One group that has high familiarity with autism is autistic adults themselves, but no study to date has examined how autistic adults form impressions of TD adults and other autistic adults.

\section{What was the purpose of this study?}

The purpose of this study was to examine whether first impressions of, and social interest in, autistic adults differ between autistic and TD raters, and determine whether disclosure of a person's diagnosis affects these patterns differently for autistic and TD raters.

\footnotetext{
${ }^{1}$ School of Behavioral and Brain Sciences, The University of Texas at Dallas, Richardson, Texas.

${ }^{2}$ nonPareil Institute, Plano, Texas.

${ }^{3}$ Department of Psychology, Southern Methodist University, Dallas, Texas.
} 
What did the researchers do?

A total of 32 TD and 32 autistic adult raters viewed videos of 40 unfamiliar adults (20 TD and 20 autistic individuals) and rated each person on six traits (awkwardness, attractiveness, assertiveness, likeability, trustworthiness, and intelligence) and four items assessing their social interest in future interaction with the person in the video. Videos were presented either with or without the person's diagnosis to see whether this information affects impressions formed by autistic and TD raters.

What were the results of the study?

Consistent with prior work, TD raters formed more negative first impressions of autistic adults than TD adults. Meanwhile, autistic raters formed more positive impressions overall, but shared the TD pattern of rating autistic adults less favorably than TD adults on several traits. However, contrary to theories about reduced social motivation in autism, autistic raters expressed greater interest than TD raters in future interaction with people in the videos and this social interest was largest for other autistic people. Finally, diagnostic disclosure improved impressions of autistic adults made by TD raters but not by autistic raters.

\section{What do these findings add to what was already known?}

These findings provide empirical evidence that autistic adults detect and interpret autistic social differences similarly to TD adults, but they express greater inclusivity and less discriminatory attitudes about these differences. These results add to a growing literature about how autistic people are perceived, how these perceptions affect their social experiences, and how similarity between social partners can support social connection.

\section{What are potential weaknesses in the study?}

The included sample was predominantly Caucasian and male, and did not include participants with intellectual disability. Future studies should examine whether the patterns here extend to more diverse samples.

How will these findings help autistic adults now or in the future?

This study provides additional evidence that social opportunities for autistic adults are affected by the perceptions and biases of potential social partners. Autistic observers may share the TD bias toward less favorable trait evaluation of autistic adults, but this did not lessen their social interest in interacting with autistic adults the way it did for TD observers. Opportunities for autistic adults to interact with other autistic adults may facilitate relationship development in this population who often struggle to have their social needs met. In addition, findings suggest that acceptance of autistic people increases with familiarity of autism itself. Improving attitudes about autism among TD people may be one effective way to improve the social experiences of autistic people within neurotypical environments.

\section{Introduction}

$\mathbf{T}$ YPICAlly DEVEloping (TD) ADUlts form unfavorable first impressions of unfamiliar autistic adults based upon brief videos of their social behavior and report reduced interest in pursuing social interaction with them. ${ }^{1-3}$ Such negative first impressions can reduce social opportunities for autistic adults, contribute to poor personal and professional outcomes, ${ }^{4}$ and affect their quality of life. ${ }^{5}$

Impressions of autistic adults, however, are quite variable across TD raters and are influenced by many factors. ${ }^{2,3}$ For instance, impressions are improved when TD adults are made aware of the autism diagnosis of the person they are evaluating. ${ }^{2}$ This may occur because awareness provides an explanation for perceived differences in social presentation styles of autistic adults, leading to more favorable judgments. Impressions are also more favorable among TD raters with higher knowledge about autism and among those who hold lower stigma toward it. ${ }^{2,3}$ These findings suggest that familiarity with autism may improve TD adults' ability to understand and accept autistic differences. ${ }^{6}$

Given these factors, first impressions of autistic adults may be expected to differ when formed by autistic raters relative to TD raters. Autistic raters have high familiarity with autism and are often more adept at inferring autistic intentions and mental states than TD individuals. ${ }^{7,8}$ The bidirectional difficulty in communication, connection, and understanding between autistic and TD populations has been called the "double empathy problem,", and is supported by growing evidence that TD people may be just as prone to misperceiving autistic people as vice versa (for a review see Milton et al. ${ }^{10}$ ). From this perspective, social interaction difficulties between autistic and nonautistic people are not reducible to a traditional social cognitive deficit model of autism (for a review of this argument, see Bottema-Beutel ${ }^{11}$ ), but instead reflect a relational disjuncture in which dispositional and neurological differences between TD and autistic people contribute to distinct social norms, expectations, and modes of understanding. ${ }^{10,12}$ 
One implication of this framework is that autistic people may demonstrate enhanced social coordination and affiliation for autistic partners during interaction relative to TD partners, ${ }^{13,14}$ a perspective that has generated some emerging empirical support. ${ }^{11,15-17}$ This study tests whether this extends to a first impression context by assessing whether autistic adults are evaluated more favorably by autistic relative to TD raters and examines whether knowledge of an autistic person's diagnosis affects evaluations differently for the two groups. Consistent with a double empathy framework, we hypothesized that autistic raters would evaluate autistic participants more favorably than would TD raters, and that they would be less affected by diagnostic disclosure because of already high familiarity with autism and its characteristics.

\section{Methods}

\section{Participants}

This study occurred in two parts. First, autistic and TD "stimulus participants" were recruited to participate in a video recording of their social behavior. Second, these videos were later evaluated by a separate group of autistic and TD "rater participants." All autistic stimulus and rater participants had confirmed diagnoses on the Autism Diagnostic Observation Schedule $^{18}$ and full-scale Intelligence Quotient (IQ) scores $>75$ as measured by the Wechsler Abbreviated Scale of Intelligence (WASI). ${ }^{19}$ Autistic participants were recruited from the student population at the University of Texas at Dallas (UTD) and from the nonPareil Institute, a nonprofit training program for autistic adults. TD stimulus participants were recruited from UTD and TD rater participants from Southern Methodist University (SMU). All participants provided informed consent, and the university institutional review board at both UTD and SMU approved the study.

Stimulus participants. Twenty autistic adults and 20 TD adults served as stimulus participants. All TD stimulus participants and 15 autistic stimulus participants were previously included as stimulus participants in Sasson et al. ${ }^{1}$ Five autistic stimulus participants from the original Sasson et al. ${ }^{1}$ study were removed and replaced with five new autistic participants because they had attended nonPareil within the previous year and would be familiar to most autistic raters. The final sample consisted of autistic $(n=20)$ and TD $(n=20)$ stimulus participants who were matched on gender (17 male, 3 female) and comparable on age $\left(M_{\text {Autistic }}=24.45\right.$ years, standard deviation $[\mathrm{SD}]=5.84 ; M_{\mathrm{TD}}=25.00$ years, $\mathrm{SD}=6.24, F(1,39)=0.083$, $p=0.78)$ and IQ $\left(M_{\text {Autistic }}=104.68, \mathrm{SD}=9.66 ; M_{\mathrm{TD}}=104.43\right.$, $\mathrm{SD}=9.13, F(1,39)=0.037, p=0.85)$ as estimated by the reading subtest of the Wide Range Achievement Test 3 (WRAT$3){ }^{20}$ Whereas autistic participants were assessed at clinical intake with the WASI to confirm eligibility for the study (full scale IQ >75), time constraints in the testing protocol required a faster estimate of IQ that could establish comparability in intellectual functioning between autistic and TD participants. The reading subtest of the WRAT-3 has been used for this purpose in prior related studies, ${ }^{1-3}$ as it correlates relatively highly with verbal and full-scale IQ. ${ }^{21}$

Rater participants. Thirty-two autistic and 32 TD adults served as raters. To best match TD and autistic raters, $84 \mathrm{TD}$ participants (34 male) were recruited from the psychology department at SMU and the 32 TD participants who most approximated the 32 autistic raters on gender, race, age, and IQ were selected. We selected TD raters first on gender and then race to match the predominantly male and Caucasian autistic rater sample. As a result, excluded TD raters were overwhelmingly female and/or not Caucasian. TD raters were recruited from SMU to ensure participants would be unfamiliar to stimulus participants recruited from UTD. Autistic raters were selected to minimize familiarity with those in the videos. Still, 12 of the 32 autistic raters recognized at least 1 of the 40 stimulus participants (mean number recognized for those 12 raters was 3.5). These cases were treated as missing data in analyses. Autistic and TD raters were majority Caucasian, and the racial composition of the two groups did not differ significantly (autistic: 28 Caucasian, 1 African American, 1 Asian, 1 Native American, 1 Other. TD: 29 Caucasian, 1 African American, 2 Asian; $\left.X^{2}(4)=0.67\right)$. Autistic and TD raters also did not significantly differ on gender (autistic: 27 male, TD: 27 male, $X^{2}(2)=1.00$ ), but did on age $\left(M_{\text {Autistic }}=24.94, \mathrm{SD}=3.74 ; M_{\mathrm{TD}}=22.16, \mathrm{SD}=3.06\right.$, $F(1,62)=8.90, p=0.004)$ and IQ estimated by the WRAT $-3^{20}$ $\left(M_{\text {Autistic }}=101.28, \quad \mathrm{SD}=13.84 ; \quad M_{\mathrm{TD}}=111.43, \quad \mathrm{SD}=9.72\right.$, $F(1,62)=10.83, p=0.002)$. Age and IQ were, therefore, covaried in data analyses.

\section{Procedures}

Stimulus participants were video recorded while completing the High-Risk Social Challenge. ${ }^{22}$ Stimulus participants spent 1 minute seated in a chair describing why they should be chosen to participate on a TV show. ${ }^{1}$ Both faces and bodies of the stimulus participants are visible in the videos, and the first 10 seconds of social behavior after impersonal introductory remarks (e.g., "okay, here I go") served as the glimpse of their social behavior later evaluated by rater participants. Any mentions by stimulus participants of their names or diagnostic status were removed with editing software. Representative examples of stimulus participant statements during these first 10 seconds include "I should be picked because I'm pretty amazing" and "I'm qualified to be on this show because I'm a frank person and am not afraid to speak my mind." A previous study by our group found that written transcriptions of autistic and TD speech during the videos are not evaluated differently by TD raters, suggesting similar conversational content between autistic and TD stimulus participants within the videos. However, TD raters did evaluate autistic people less favorably when visual and/or auditory information were provided, including when only a static image was presented. ${ }^{1}$

This task was chosen because it represents a compromise between experimental control and ecological validity (i.e., how social behavior occurs in the real world). By ensuring that all participants are recorded under the same scenario, any rating differences that occur for the autistic and TD groups cannot be attributed to differences in the social conditions or expectations under which they were recorded. At the same time, unlike some prior studies in which perceptions of autistic people have been examined using vignettes or actor portrayals of autism, ${ }^{23,24}$ the task used here captures authentic behavior of autistic and nonautistic people within conditions similar to those in which first impressions are often formed (see Sasson et al. ${ }^{1}$ for more details of stimulus creation). 
Next, a separate group of autistic and TD raters viewed the 40 stimulus videos one at a time in a randomized order. Half of the videos were presented with an accurate diagnostic label (i.e., autistic adults as "this person has autism" and TD adults as "this person has no diagnosis") and half were presented with no label. An alternative version of the task was created that reversed which videos were labeled and which were unlabeled, and all raters were randomly assigned to one of the two versions. After viewing each video, raters provided their first impression of each stimulus participant by completing the First Impressions Scale ${ }^{1}$ that contains 10 items evaluating the rater's impressions of the stimulus participant. The scale includes six trait items (awkwardness, attractiveness, trustworthiness, intelligence, assertiveness, and likability), and four behavioral intent items (e.g., I would sit next to this person) rated on a 4-point scale that ranges from strongly disagree to strongly agree. All items were scored such that higher ratings indicated more favorable impressions, and each item was assessed separately in analyses. The internal consistency of the first impression scale was good (Cronbach's $\alpha=0.79$ ).

\section{Analytic plan}

Cross-classified random effects models were run for each first impression scale item using multilevel modeling with Restricted Maximum Likelihood estimation and variance components covariance structure. Effects were estimated for stimulus participant diagnosis (autistic or TD), rater diagnosis (autistic or TD), and stimulus participant label condition (diagnostic label or no label), and interaction terms were created to examine moderated effects. Significant interaction terms were followed up with simple slopes by dummy coding categorical variables and examining at what level the slope was significant. Rater participant age, gender, and IQ were entered as covariates, categorical predictors were effects coded, and all continuous predictors were grand mean centered.

\section{Results}

Means for all first impression ratings in each condition are displayed in Figure 1. There were significant main effects for rater diagnosis, stimulus participant diagnosis, and label. First, autistic stimulus participants were evaluated less favorably than TD stimulus participants. They were rated as more awkward, less attractive, and less dominant ( $p$ 's $>0.001$ ), and raters reported lower desire to hang out with, sit near, and have a conversation with them ( $p$ 's $>0.001)$. Second, autistic raters evaluated stimulus participants more favorably than TD raters, rating them as more attractive $(p=0.018)$ and reported more interest in hanging out with them $(p=0.002)$. Third, stimulus participants with diagnostic labels were evaluated more positively than when no label was provided. They were rated as more likeable $(p=0.002)$ and trustworthy $(p=0.001)$, and raters reported more interest in hanging out with $(p=0.006)$ and having a conversation $(p=0.015)$ with them. There were no other effects of stimulus participant diagnosis ( $p$ 's $>0.08)$, rater diagnosis ( $p$ 's $>0.16$ ), or label ( $p$ 's $>0.065)$.

We next examined the two-way interaction of rater diagnosis with stimulus participant diagnosis to test the hypothesis that TD and autistic rater evaluations would depend upon the diagnosis of the stimulus participant (Table 1). Significant interactions and simple slopes were found for
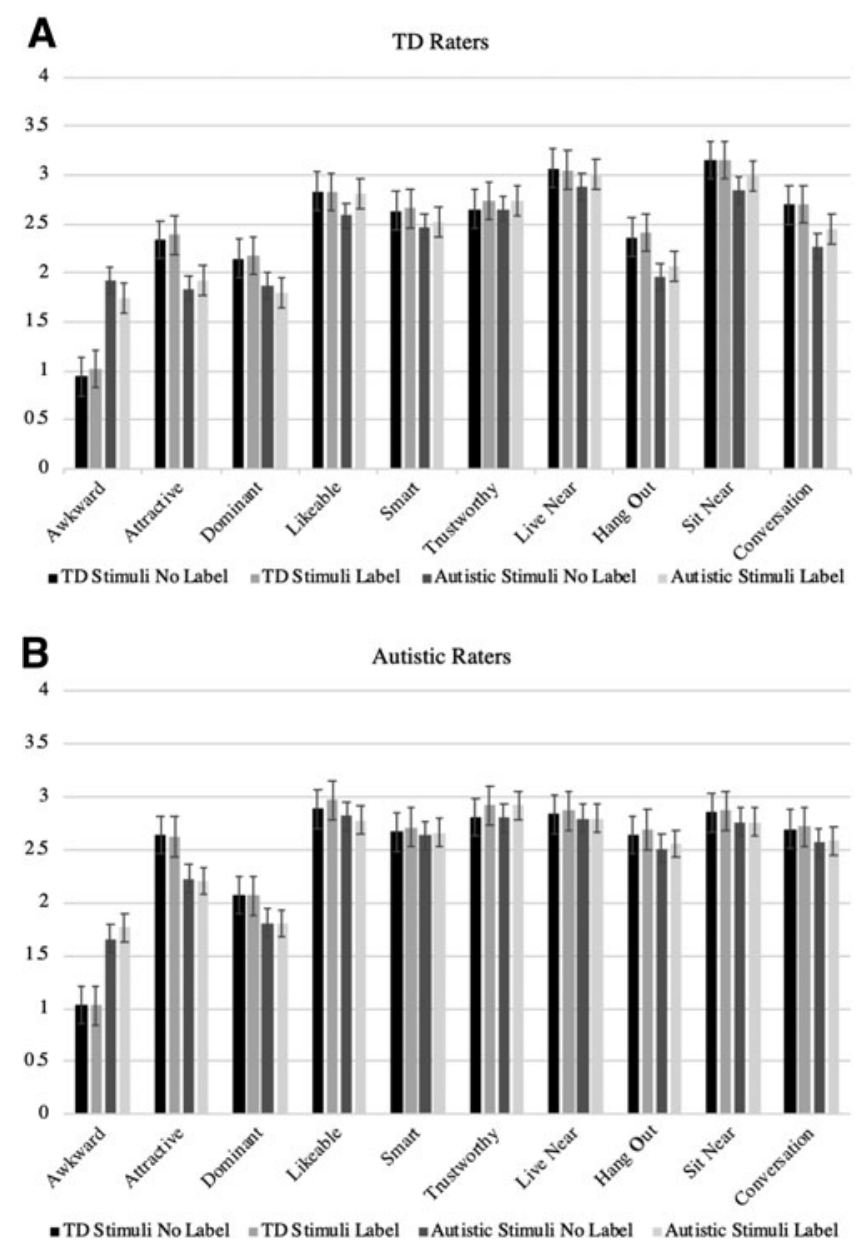

FIG. 1. (A, B) Mean ratings of first impressions for combinations of stimulus participant and rater diagnosis and labeling condition. Error bars indicate standard error. TD, typically developing.

ratings of hanging out with and sitting near ( $p$ 's $<0.01)$. Autistic raters reported more interest than TD raters in hanging out with stimulus participants, and this effect was strongest for ratings of autistic stimulus participants (autistic stimulus participants: $b=0.26$, standard error $[\mathrm{SE}]=0.06$, $p<0.001, d=0.99$; TD stimulus participants: $b=0.14$, $\mathrm{SE}=0.06, p=0.027, d=0.54)$. Furthermore, TD raters were more comfortable than autistic raters in sitting near TD stimulus participants $(b=-0.14, \mathrm{SE}=0.06, p=0.024 ; d=0.57)$, but there were no differences in desire to sit near autistic stimulus participants $(b=-0.08, \mathrm{SE}=0.06, p=0.21 ; d=0.32$ ). Interaction terms were also significant for ratings of awkwardness, intelligence, and having a conversation with $(p$ 's $<0.02)$. Although these terms indicate significant differences in how stimulus participants are evaluated by raters, simple slopes follow-ups indicated the simple coefficients did not differ from zero. Autistic raters evaluated TD stimulus participants as less awkward and autistic stimulus participants more awkward than TD raters (TD stimulus participant: $b=-0.03, \mathrm{SE}=0.05, p=0.622$; autistic stimulus participant: $b=0.06, \mathrm{SE}=0.06, p=0.24 ; d=0.19)$. Autistic raters also evaluated TD and autistic stimulus participants as more intelligent (TD stimulus participants: $b=0.02, \mathrm{SE}=0.07$, 


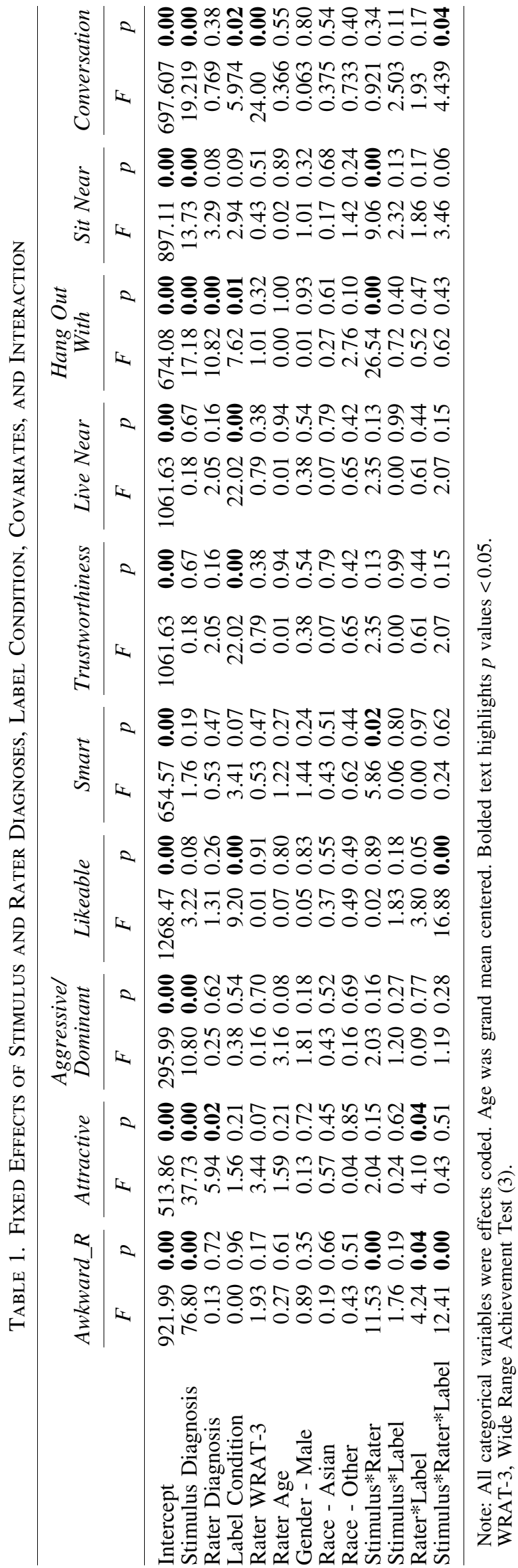

$p=0.74$; autistic stimulus participants: $b=0.07, \mathrm{SE}=0.07$, $p=0.28 ; d=0.17$, and reported more interest in having a conversation with the stimulus participant (TD stimulus participants: $b=0.0003, \mathrm{SE}=0.06, \quad p=0.996 ;$ autistic stimulus participants $b=0.11, \mathrm{SE}=0.06, p=0.09 ; d=0.23$ ) compared with TD raters, with these effects being strongest for ratings of autistic stimulus participants.

Although some significant two-way interactions of label emerged with both stimulus participant diagnosis and rater diagnosis, these were subsumed by a three-way interaction between stimulus participant diagnosis, rater diagnosis, and label (Table 1). This three-way interaction examined whether TD and autistic rater evaluations would depend upon whether a diagnostic label was included for autistic and TD stimulus participants. Significant effects were found for ratings of awkwardness, likeability, and interest living near and having a conversation with $(p$ 's $<0.045)$. Breaking down these interactions revealed significant two-way interactions between rater diagnosis and label for autistic stimulus participants $(p$ 's $<0.05)$ but not for TD stimulus participants ( $p$ 's $>0.124)$; thus, only effects for autistic stimulus participants were followed up. TD raters evaluated autistic stimulus participants as less awkward when they were labeled as having autism compared with when no label was provided $(b=0.09, \mathrm{SE}=0.03, p<0.001 ; d=0.34)$, but the opposite pattern occurred for autistic raters: they rated autistic stimulus participants as being less awkward when they were given no label compared with when a label of autism was provided $(b=-0.05, \mathrm{SE}=0.03, p=0.039 ; d=0.20)$. TD raters also evaluated autistic stimulus participants as more likeable $(b=0.11, \mathrm{SE}=0.02, p<0.001 ; d=0.49)$ and had a stronger desire to live near them $(b=0.06, \mathrm{SE}=0.02, p=0.004$; $d=0.22)$ and have a conversation with them $(b=0.08$, $\mathrm{SE}=0.02, p<0.001 ; d=0.22)$ when they were labeled as having autism compared with when no label was provided, but these effects did not occur for autistic raters, whose ratings on these items did not differ when an autism label was included compared with when it was withheld ( $p$ 's $>0.41$ ).

\section{Discussion}

This study examined whether autistic and TD adults differ in their first impressions of autistic adults, and whether diagnostic disclosure affects impression formation differently in the two groups. Consistent with prior work, ${ }^{1,2}$ TD raters evaluated autistic adults less favorably than TD controls. Autistic raters were more favorable than TD raters overall, rating people as more attractive and expressing more interest in hanging out with them, but like their TD counterparts, they evaluated autistic adults less favorably than TD adults on several traits, judging them to be more awkward, less attractive, and less dominant than TD controls.

Such findings are inconsistent with social cognitive deficit interpretations of autistic social evaluation. In this study, autistic raters not only detected social presentation differences displayed by autistic adults, but they also formed similar evaluative judgments about them relative to their TD counterparts, a similar pattern to what has recently been found with autistic adolescents. ${ }^{25}$ Thus, despite autism in adulthood being characterized by poorer social cognitive performance on many standardized assessments, ${ }^{26}$ this did not translate to social evaluation differences within a first 
impression context. In contrast to a social attunement hypothesis $^{27}$ that posits that atypical social expression in autistic adults may, in part, derive from a difficulty perceiving and emulating socially normative standards, autistic raters in this study were just as sensitive as TD raters to social presentation differences in autistic adults and evaluated them similarly. Such evidence implies that social presentation differences in autism are likely not driven by a lack of ability in detecting and interpreting normative social signifiers.

It also suggests that, contrary to hypotheses, simply being autistic themselves and having greater familiarity and experiences with autism does not result in more favorable impressions of other autistic adults. Instead, this may indicate that they share the TD negativity bias toward autistic social presentations, perhaps reflecting the internalization of broader cultural norms that perceive such autistic differences less favorably. Given that similar patterns have also been found during adolescence, ${ }^{25}$ future studies are encouraged to examine whether they extend to the same degree to younger children. If unfavorable impressions of autistic people only emerge later in development, this may suggest that TD and autistic individuals are socialized into these negative evaluations. If this is the case, stigma toward autism by TD individuals, ${ }^{28}$ and the internalized stigma that occurs for some autistic adults, ${ }^{29}$ may reflect the developmental acquisition of implicit and explicit cultural attitudes about autism. To counteract this process, greater societal inclusion and integration of autistic people from a very early age may be helpful. Meaningful intergroup contact has been found to reduce stigma, prejudice, and biases for other marginalized groups, ${ }^{30}$ and this process might also be expected to occur for autism. In turn, improving attitudes about autism and reducing stigma should enhance the social experiences of autistic people and better enable them to meet their personal and professional goals.

Furthermore, in contrast to theories about reduced social motivation in autism, ${ }^{31}$ autistic raters expressed greater interest than TD raters in hanging out with stimulus participants, and this effect was largest when the stimulus participants were autistic. The latter finding aligns with a double empathy framework of autism, ${ }^{9}$ which posits that social connection and intersubjectivity are facilitated by neurologic and experiential similarity. In this study, despite sharing the TD tendency to evaluate autistic adults less positively, autistic raters do not appear to judge these traits as an impediment to social interaction in the same way that their TD counterparts do. Rather, autistic raters may be detecting similarities between themselves and the autistic stimulus participants that result in a stronger desire to interact with them. ${ }^{32}$ Given the heightened social interest expressed here by the autistic raters, these findings suggest that increasing opportunities for autistic adults to interact with other autistic adults, including social programs that connect autistic adults with each other, may facilitate the formation of friendships or other relationships for autistic adults who often struggle to have their social needs met. ${ }^{33}$

Consistent with prior findings, ${ }^{2,3}$ awareness of the stimulus participants' diagnosis improved TD impressions of autistic adults. This pattern, however, did not occur for autistic raters. Whereas TD raters became less judgmental when they were provided a diagnosis, likely because it offered an explanation for the social presentation differences they tended to interpret negatively, autistic raters either already inferred the diagnostic status of autistic adults when a label was absent, thereby not shifting their impressions when it was included, or for them the diagnosis is simply less relevant in terms of impression formation. There was, however, one exception to this interpretation. Autistic raters actually evaluated autistic adults as more awkward when their diagnosis was provided relative to when it was withheld. It may be the case that autistic raters are highly cognizant and sensitive about autism being associated with awkwardness, and providing a diagnostic label increased the salience of such indicators in autistic stimulus participants. This may be why other traits less specifically associated with autism (trustworthiness, intelligence, etc.) did not produce a similar effect. Perceptions of awkwardness may be particularly salient to autistic adults, who are more aware than others about autistic traits and how they can be judged and stigmatized, 5,34 leading many to partake in effortful masking behaviors to avoid negative responses. $^{35}$

Several limitations should be considered when interpreting the results reported in this study. First, the sample here largely consisted of autistic adults who were male, Caucasian, and scored in the average to above average range on tests of cognitive ability. Findings may have differed with a more diverse sample of raters and stimulus participants. In particular, autistic women may be more motivated and successful at masking their autistic behaviors, ${ }^{35,36}$ and thus inclusion of more autistic women as stimulus participants may have produced different effects for autistic women compared with men, as has been shown in one recent study. ${ }^{37}$ Second, a larger sample may have produced increased power to detect more nuanced and targeted effects (e.g., three-way interactions) and would have enabled assessment of possible measurement invariance (i.e., analyzing whether autistic and TD raters interpreted items the same way). Furthermore, analyses included multiple comparisons that raise the possibility of type I error, and some of the effect sizes of some of the significant results were small. Caution should be used when interpreting these particular findings. Finally, to prevent familiarity with stimulus participants, TD raters were recruited from a separate university and this resulted in a TD rater group that was matched with the autistic rater group on gender and race but differed slightly on age and IQ. Although these differences were covaried in all analyses and, therefore, did not affect reported results, there may be other unaccounted for differences between the groups that may have influenced ratings.

Despite these limitations, this study advances our understanding of perceptions of autistic adults in several important ways. First, autistic adults appear to form first impressions in largely similar ways to TD adults. Second, despite sharing the TD negativity bias toward trait evaluation of autistic adults, autistic raters express greater interest than their TD counterparts in socially interacting with autistic adults, supporting a double empathy perspective on autistic sociality. ${ }^{9}$ Finally, diagnostic disclosure resulted in more positive impressions of autistic adults for TD raters but not for autistic raters, suggesting distinct effects of diagnostic awareness in the two groups. Collectively, findings from this study suggest that autistic adults detect and evaluate autistic social presentation differences similarly to TD adults, but these less favorable trait judgments do not diminish their interest in potential 
social interaction with autistic individuals as it does for their TD counterparts. Autistic adults may, therefore, find more receptive social partners in, and form more positive affiliations with, other autistic adults relative to TD partners.

\section{Acknowledgments}

The authors thank Isabel Magana, Malina Maharana, Matti Miller, David Rachman, Steven Raveneau, Hulon Sherard, and Mayson Trujillo for their assistance with data collection.

\section{Authors' Contributions}

K.M.D. as the lead author, contributed to the design, data collection, and writing of the article. K.E.M. also contributed to the design, data collection, analysis, and assisted with writing. D.R.J. assisted with data collection and design. D.J.F. contributed to study design, data collection, and interpretation of the results. M.C. aided with data collection and writing. N.J.S. oversaw study design, data collection, data analysis, and writing of the article. All coauthors have reviewed and approved this article prior to submission.

\section{Author Disclosure Statement}

No authors have any competing financial conflicts of interest to disclose.

\section{Funding Information}

This research was supported by internal institutional funds provided to N.J.S.

\section{References}

1. Sasson NJ, Faso DJ, Nugent J, Lovell S, Kennedy DP, Grossman RB. Neurotypical peers are less willing to interact with those with autism based on thin slice judgments. Sci Rep. 2017;7:1-10.

2. Sasson NJ, Morrison KE. First impressions of adults with autism improve with diagnostic disclosure and increased autism knowledge of peers. Autism. 2019;23(1):50-59.

3. Morrison KE, DeBrabander KM, Faso DJ, Sasson NJ. Variability in first impressions of autistic adults made by neurotypical raters is driven more by characteristics of the rater than by characteristics of autistic adults. Autism. 2019 [Epub ahead of print]; https://doi.org/10.1177/1362361318824104.

4. Belch HA. Retention and students with disabilities. J Coll Student Retent Res Theory Pract. 2004;6(1):3-22.

5. Cage E, Di Monaco J, Newell V. Experiences of autism acceptance and mental health in autistic adults. J Autism Dev Disord. 2018;48(2):473-484.

6. Gardiner E, Iarocci G. Students with autism spectrum disorder in the university context: Peer acceptance predicts intention to volunteer. J Autism Dev Disord. 2014;44(5): 1008-1017.

7. Heasman B, Gillespie A. Perspective-taking is two-sided: Misunderstandings between people with Asperger's syndrome and their family members. Autism. 2018;22(6): 740-750.

8. Sheppard E, Pillai D, Wong GTL, Ropar D, Mitchell P. How easy is it to read the minds of people with autism spectrum disorder? J Autism Dev Disord. 2016;46(4): $1247-1254$.
9. Milton DEM. On the ontological status of autism: The "double empathy problem." Disabil Soc. 2012;27(6): 883-887.

10. Milton DEM, Heasman B, Sheppard E. Double empathy. In: Volkmar F, ed. Encyclopedia of Autism Spectrum Disorders. New York, NY: Springer; 2018;1-8.

11. Bottema-Beutel K. Glimpses into the blind spot: Social interaction and autism. J Commun Disord. 2017;68:24-34.

12. Nicolaidis C, Milton D, Sasson NJ, Sheppard E (Lizzy), Yergeau M. An expert discussion on autism and empathy. Autism Adulthood. 2019;1(1):4-11.

13. Beardon L. Autism and Asperger Syndrome in Adults. London: Sheldon Press; 2017.

14. Chown N. More on the ontological status of autism and double empathy. Disabil Soc. 2014;29(10):1672-1676.

15. Heasman B, Gillespie A. Neurodivergent intersubjectivity: Distinctive features of how autistic people create shared understanding. Autism. 2018;23(4):910-921.

16. Gernsbacher MA, Stevenson JL, Dern S. Specificity, contexts, and reference groups matter when assessing autistic traits. PLoS One. 2017;12(2):e0171931.

17. Crompton CJ, Fletcher-Watson S. Efficiency and interaction during information transfer between autistic and neurotypical people. Presented at International Society for Autism Research Annual Meeting, Montreal, Canada, May $1-4,2019$.

18. Lord C, Risi S, Lambrecht L, et al. The Autism Diagnostic Observation Schedule-Generic: A standard measure of social and communication deficits associated with the spectrum of autism. J Autism Dev Disord. 2000;30(3):205223.

19. Wechsler D. Wechsler Abbreviated Scale of IntelligenceSecond Edition (WASI-II). San Antonio, TX: NCS Pearson; 2011.

20. Wilkinson G. Wide Range Achievement Test WRAT. Wilmington, DE: Wide Range Inc.; 1993.

21. Kareken DA, Gur RC, Saykin AJ. Reading on the Wide Range Achievement Test-Revised and parental education as predictors of IQ: Comparison with the Barona formula. Arch Clin Neuropsychol. 1995;10(2):147-57.

22. Gibson CM, Penn DL, Prinstein MJ, Perkins DO, Belger A. Social skill and social cognition in adolescents at genetic risk for psychosis. Schizophr Res. 2010;122(1-3): 179-184.

23. Matthews NL, Ly AR, Goldberg WA. College students' perceptions of peers with autism spectrum disorder. $J$ Autism Dev Disord. 2014;45(1):90-99.

24. Nevill REA, White SW. College students' openness toward autism spectrum disorders: Improving peer acceptance. $J$ Autism Dev Disord. 2011;41(12):1619-1628.

25. Grossman RB, Mertens J, Zane E. Perceptions of self and other: Social judgments and gaze patterns to videos of adolescents with and without autism spectrum disorder. Autism. 2019;23(4):846-857.

26. Morrison KE, Pinkham AE, Kelsven S, Ludwig K, Penn DL, Sasson NJ. Psychometric evaluation of social cognitive measures for adults with autism. Autism Res. 2019;12(5): 766-778.

27. Shriberg LD, Paul R, Black LM, Van Santen JP. The hypothesis of apraxia of speech in children with autism spectrum disorder. J Autism Dev Disord. 2011;41(4):405426.

28. Gillespie-Lynch K, Brooks PJ, Someki F, et al. Changing college students' conceptions of autism: An online training 
to increase knowledge and decrease stigma. J Autism Dev Disord. 2015;45(8):2553-2566.

29. Bachmann CJ, Höfer J, Kamp-Becker I, et al. Internalised stigma in adults with autism: A German multi-center survey. Psychiatry Res. 2019;276:94-99.

30. Pettigrew TF, Tropp LR. How does intergroup contact reduce prejudice? Meta-analytic tests of three mediators. Eur J Soc Psychol. 2008;38(6):922-934.

31. Chevallier C, Kohls G, Troiani V, Brodkin ES, Schultz RT. The social motivation theory of autism. Trends Cogn Sci. 2012;16(4):231-239.

32. Montoya RM, Horton RS, Kirchner J. Is actual similarity necessary for attraction? A meta-analysis of actual and perceived similarity. J Soc Pers Relat. 2008;25:889-923.

33. Billstedt E, Gillberg C, Gillberg C. Autism after adolescence: Population-based 13- to 22-year follow-up study of 120 individuals with autism diagnosed in childhood. $J$ Autism Dev Disord. 2005;35(3):351-360.

34. Gillespie-Lynch K, Kapp SK, Brooks PJ, Pickens J, Schwartzman B. Whose expertise is it? Evidence for au- tistic adults as critical autism experts. Front Psychol. 2017; 8(MAR):1-14.

35. Hull L, Petrides KV, Allison C, et al. "Putting on my best normal": Social camouflaging in adults with autism spectrum conditions. J Autism Dev Disord. 2017;47(8): 2519-2534.

36. Lai MC, Lombardo MV, Ruigrok ANV, et al. Quantifying and exploring camouflaging in men and women with autism. Autism. 2017;21(6):690-702.

37. Cage E, Burton H. Gender differences in the first impressions of autistic adults. PsyArXiv. 2019:1-32.

Address correspondence to: Kilee M. DeBrabander, BS School of Behavioral and Brain Sciences The University of Texas at Dallas 800 West Campbell Road Richardson, TX 75080

Email: kilee.debrabander@utdallas.edu 